\title{
MONTE CARLO SIMULATION OF A WHOLE-BODY COUNTER USING IGOR PHANTOMS
}

\author{
François O. Bochud ${ }^{1, *}$, Jean-Pascal Laedermannn ${ }^{1}$, Sébastien Baechler ${ }^{1}$, Claude J. Bailat ${ }^{1}$, Markus Boschung ${ }^{2}$, \\ Abbas Aroua ${ }^{1}$ and Sabine Mayer ${ }^{2}$ \\ ${ }^{1}$ Institute of Radiation Physics (IRA), Lausanne University Hospital, Lausanne, Switzerland \\ ${ }^{2}$ Division for Radiation Safety and Security, Paul Scherrer Institute (PSI), Villigen PSI, Switzerland \\ *Corresponding author: francois.bochud@chuv.ch
}

Received 12 September 2013; revised 20 November 2013; accepted 23 November 2013

\begin{abstract}
Whole-body counting is a technique of choice for assessing the intake of gamma-emitting radionuclides. An appropriate calibration is necessary, which is done either by experimental measurement or by Monte Carlo (MC) calculation. The aim of this work was to validate a MC model for calibrating whole-body counters (WBCs) by comparing the results of computations with measurements performed on an anthropomorphic phantom and to investigate the effect of a change in phantom's position on the WBC counting sensitivity. GEANT MC code was used for the calculations, and an IGOR phantom loaded with several types of radionuclides was used for the experimental measurements. The results show a reasonable agreement between measurements and MC computation. A 1-cm error in phantom positioning changes the activity estimation by $>2 \%$. Considering that a 5-cm deviation of the positioning of the phantom may occur in a realistic counting scenario, this implies that the uncertainty of the activity measured by a WBC is $\sim 10-20 \%$.
\end{abstract}

\section{INTRODUCTION}

Whole-body counting is a well-established technique in health physics that has been used for decades to identify and quantify gamma-emitting radioisotopes in the human body. Its principle is to scan the human body using a gamma detector (scintillation and/or semiconductor detector). Alpha- and beta-emitters are more difficult to measure with a whole-body counter (WBC) and usually require the measurement of a biological sample (urine, faeces and blood). Various WBC settings are used for measurement: bed (lying), chair (sitting) and vertical (standing). The strength of the WBC lays in its ability to provide direct measurement of intake, but in order to provide accurate results, the device must be properly characterised.

WBCs are usually calibrated using a phantom that simulates the human body, containing known radioactive substances with known activity distributions and concentrations. The calibration may be performed by measuring physical phantoms, such as the RANDO phantom developed by Alderson et al. ${ }^{(1)}$, the LLNL phantom developed at the Lawrence Livermore National Laboratory ${ }^{(2)}$, the BOttle Manikin ABsorber (BOMAB) phantom, defined by the American National Standards Institute ${ }^{(3)}$, the IGOR phantom developed at St Petersburg ${ }^{(4)}$, the Bottle-shaped phantom developed at the University Hospital of Cologne ${ }^{(5)}$ or the Block-shaped phantom developed at the Japan Atomic Energy Research Institute ${ }^{(6)}$. The calibration may also be undertaken by computation (virtual calibration) using either mathematical models simulating physical phantoms or virtual computational models such as the first stylised phantoms developed at the Oak Ridge National Laboratory based on recommendations of the Medical Internal Radiation Dose Committee $^{(7,8)}$ and the ADAM and EVA phantoms developed at Helmholtz Zentrum München ${ }^{(9)}$. These phantoms are based on simple mathematical equations (quadratic) simulating simple shapes as rough approximations of the human organs and tissues. Voxel phantoms, based on CT or MRI anatomical images of the human body, are becoming the reference ${ }^{(10)}$ and are recommended by ICRP ${ }^{(11)}$. Many phantoms have been developed in the last decade with a resolution ranging from $\sim 2$ million to 6 billion voxels, some of them related to Caucasian populations and others to non-Caucasian ones (Japanese, Chinese and Korean) ${ }^{(12-18)}$.

Other techniques and advanced mathematics are used today to develop phantoms that are race specific, such as statistical shape modelling ${ }^{(19)}$, and phantoms with smoothed organ surface contours such as the 'Non Uniform Rational B-Spline' shaping spline function $^{(20,21)}$ and polygon meshes ${ }^{(22,23)}$. A good review of physical and computational phantoms is done by the Consortium of Computational Human Phantoms ${ }^{(24)}$.

Mathematical phantoms allow for a wide range of investigations and sensitivity analysis on the various WBC settings, by means of Monte Carlo (MC) calculations. Various MC computer codes have been used so far: 'FLUktuierende KAskade' (FLUKA) ${ }^{(25)}$, 'Monte 
Carlo N-Particle' (MCNP) ${ }^{(26-33)}$, 'Electron Gamma Shower' (EGS) $)^{(5,6,34,35)}$, 'PENetration and Energy LOss of Positrons and Electrons' (PENELOPE) ${ }^{(32)}$, 'GEometry ANd Tracking' (GEANT) ${ }^{(36)}$ or local codes developed ad hoc ${ }^{(37)}$. MC calculation has been used to compare different mathematical phantoms used in $\mathrm{WBCs}^{(10,26,27,29,31,35,38)}$ or to compare the measurements obtained with a physical phantom with the results obtained by a simulated version of this phantom ${ }^{(5,6,30,35,37)}$. MC calculations are also used to investigate how the counting efficiency of the WCB depends on various parameters of the system that may be sources of uncertainty, such as the difference between the shape and size of the person to be monitored and the phantom, the measuring geometry, notably the position of the detector, the phantomdetector distance, the distribution of the radionuclides in the body, etc. ${ }^{(5,29,33,37,39)}$

The objects of the work presented in this paper are as follows: (a) validating a MC simulation used to investigate the response of a WBC with an IGOR phantom, by comparing the results of the simulation with experimental measurements, (b) performing a monoenergetic calibration of the WBC, with the possibility of extending the calibration to any other gamma emitter and (c) estimating the role of phantom positioning and explore its effect on the response of the WBC.

\section{MATERIAL AND METHODS}

\section{WBC setting}

The Paul Scherrer Institute (PSI) at Villigen (Switzerland) runs a WBC set-up that monitors a few hundred professionally exposed radiation workers per year $\left(269\right.$ in $\left.2011^{(40)}\right)$ for intake of radionuclides. The PSI-WBC is placed in a steel room, with internal dimensions of $270 \times 190 \times 220 \mathrm{~cm}$, located underground $^{(41)}$. The thickness of the steel walls, roof and base is $18 \mathrm{~cm}$; the walls are covered with $3 \mathrm{~mm}$ of lead to absorb scattered and characteristic radiation. The dose-rate level in the room amounts to $24 \mathrm{nSv} \mathrm{h}^{-1}$. As shown in Figure 1, the PSI-WBC is equipped with a detector mounted on a mechanical support that is fixed on the ceiling and which permits movements in three directions and turns around two axes; the detector's inclination is also adjustable. The dentist's chair used allows for sitting and lying positioning.

\section{Detector}

The detector used is a p-type high-purity germanium (HPGe) coaxial detector manufactured by Canberra (former: Eurysis Mesures), EGPC 100-225-R type, with a crystal diameter of $80.4 \mathrm{~mm}$ and length of 83.8 $\mathrm{mm}$ and a sensitive volume of $413 \mathrm{~cm}^{3}$. The detector measures photons with energies ranging between 50

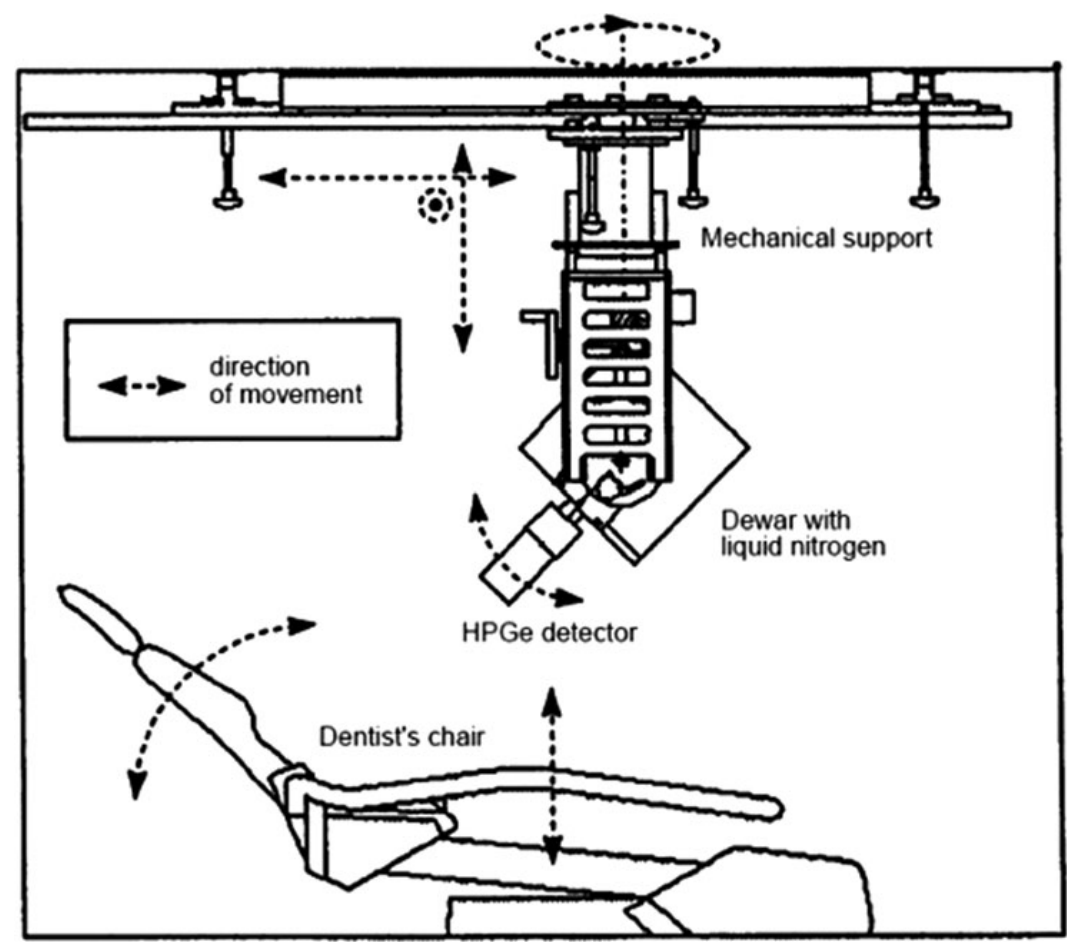

Figure 1. The PSI-WBC setting. The directions for movement are specified with dotted lines and arrows ${ }^{(41)}$. 


\section{F. O. BOCHUD ET AL.}

$\mathrm{keV}$ and $2 \mathrm{MeV}$, with an energy resolution of $2.25 \mathrm{keV}$ at $1.33 \mathrm{MeV}$. It has been fully characterised in a previous study ${ }^{(42)}$.

\section{IGOR phantom}

The IGOR phantom, also called a unified phantom, is used in Switzerland as a reference physical phantom. It was developed at the Research Institute for Industrial and Marine Medicine and the Research and Technical Centre Protection, St Petersburg( ${ }^{(4)}$. The IGOR phantom consists of polyethylene blocks (density, $0.95 \mathrm{~g} \mathrm{~cm}^{-3}$ ) of two dimensions: big size $(165 \times 110 \times 55 \mathrm{~mm} ; 0.88 \mathrm{~kg})$ and small size $(165 \times$ $110 \times 25 \mathrm{~mm} ; 0.40 \mathrm{~kg}$ ). As shown in Table 1 , six configurations are possible with the IGOR phantom; they are referred to as P1 to P6 and correspond, respectively, to a toddler, a child, a teenager and an adult of three different weights $(70,90$ and $110 \mathrm{~kg})$. Figure 2 shows the IGOR phantom (configuration P2) posed on the dentist's chair in a sitting position. In this study, the authors used five phantom configurations (P2 to $\mathrm{P} 6$ ).

\section{Radioactive sources}

Each block of the phantom is equipped with two cylindrical holes to host rods of $6 \mathrm{~mm}$ diameter and 163 $\mathrm{mm}$ length containing the radioactive substance. Experimental measurements with the various configurations of the IGOR phantom were made at the PSI for the five radionuclides: Eu-152, Ba-133, Co-60, Cs137 and $\mathrm{K}-40^{(43)}$. This covers a range of energies from 81 to $1461 \mathrm{keV}$ as shown in Table 2, which also gives the activities used.

In a number of cases, the radioactive rods available were not sufficient to fill all the blocks of the phantom. These cases are presented in Table 3.

\section{Monte Carlo code}

The MC code used in this investigation was the last version of GEANT (4.9.5.p01) developed at $\mathrm{CERN}^{(44,45)}$. This version is provided with three models for simulating low-energy electromagnetic radiation: 'Standard', 'Penelope' and 'Livermore'. They only differ for energies of $<30 \mathrm{keV}$. The authors chose to use 'Penelope', but preliminary tests did not find a strong effect on the models. $\mathrm{A} \mathrm{C}++$ implementation of the nuclide decay scheme simulator SCH2FOR was used ${ }^{(46)}$. The computer cluster of the Institute of Radiation Physics, Lausanne, was used for computation; it consists of 12 PC $2.8 \mathrm{MHz}$ bioprocessors, under Linux Ubuntu 10.04 LTS. The GEANT simulations were run in parallel; the openMPI software was used to this effect. The number of histories was chosen to obtain a statistical uncertainty on the order of $1 \%$, leading to several hundred million histories and a few tens of days of computations for the various configurations (five phantoms, five radionuclides and monoenergetic photons).

\section{Statistical significance}

If the measured sensitivity, or counting efficiency, $\varepsilon_{\mathrm{M}}$, is given with an absolute standard uncertainty $u_{\mathrm{M}}$ and the MC-calculated sensitivity $\varepsilon_{\mathrm{C}}$ is given with Poisson statistics uncertainty $u_{\mathrm{C}}$, the relative bias relbias and the $z$-score are defined as follows:

$$
\begin{aligned}
& \text { rel-bias }=\frac{e_{\mathrm{C}}}{e_{\mathrm{M}}}-1, \\
& z \text {-score }=\frac{e_{\mathrm{C}}-e_{\mathrm{M}}{ }^{1 / 2}}{u_{\mathrm{C}}^{2}+u_{\mathrm{M}}{ }^{2}} .
\end{aligned}
$$

The sensitivity $\varepsilon$, also called peak efficiency, is expressed as the integral number of counts in the peak per second, divided by the activity of the nuclide in becquerel, multiplied by the emission probability.

Under generally accepted standard conditions, this $z$-score behaves like a normal reduced random variable, so a set of non-significant independent $z$-scores should contain $\sim 95 \%$ of the values in the interval $[-2,2]$, and a value outside this interval will indicate a statistically significant difference. To compare sets of independent values, the fact that the quadratic sum of $n z$-scores behaves like a chi-square random variable of $n$ degrees of freedom is assumed. The

Table 1. Anthropometric parameters of the various configurations of the IGOR phantom.

\begin{tabular}{llccrrr}
\hline Phantom & \multicolumn{1}{c}{ Simulation } & Weight $(\mathrm{kg})$ & Height $(\mathrm{cm})$ & $\begin{array}{c}\text { Average } \\
\text { thickness }(\mathrm{cm})\end{array}$ & $\begin{array}{c}\text { Number of } \\
\text { big blocks }\end{array}$ & $\begin{array}{c}\text { Number of } \\
\text { small blocks }\end{array}$ \\
\hline P1 & Toddler (2 y, 12 kg) & 10.6 & 82.5 & 8.8 & 12 & 0 \\
P2 & Child (6 y, 24 kg) & 20.9 & 121.0 & 10.9 & 11.8 & 36 \\
P3 & Teenager (14 y, 50 kg) & 42.9 & 160.0 & 14.3 & 69 & 28 \\
P4 & Adult (70 kg) & 61.5 & 170.5 & 15.7 & 72 & 36 \\
P5 & Adult (90 kg) & 77.8 & 170.5 & 19.4 & 90 & 40 \\
P6 & Adult (110 kg) & 95.2 & 170.5 & & & \\
\hline
\end{tabular}


significance will be a $p$-value associated with the computed chi-square. A $p$-value of $>5 \%$ will be considered as non-significant.

\section{Validation of the Monte Carlo code}

Various preliminary checks were undertaken. First, the simulations were performed on three types of 'phantoms' (a single polyethylene IGOR block, six blocks and the regular P4 phantom) successively filled with a given nuclide (Ba-133, Cs-137 and Co-60) using two MC codes: GEANT4 and MCNPX. This was repeated for three different positions of the detector in order to compare the counting efficiency calculated by each MC code.

After this, the authors only used the GEANT4 code and explored to what degree the use of radioactive rods is representative of a homogeneous distribution of the activity within the phantom. This was also done with the $\mathrm{P} 4$ phantom.

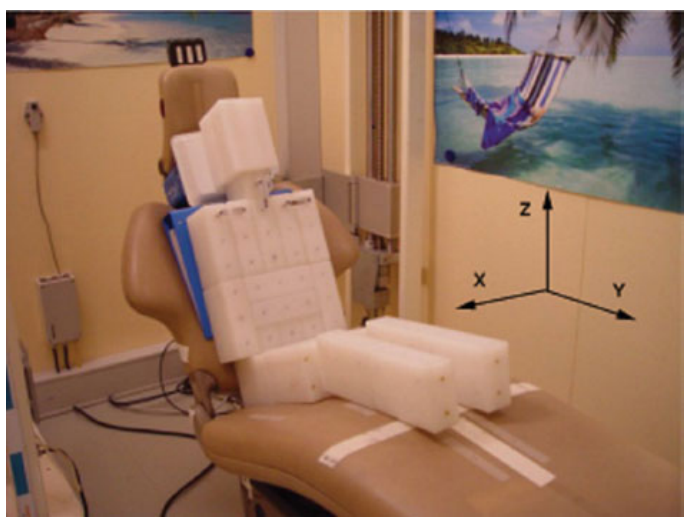

Figure 2. IGOR P2 configuration corresponding to a $6-y-$ old child ${ }^{(43)}$.

\section{Monte Carlo simulations}

The various experimental settings related to the PSIWBC and the different configurations of the IGOR phantom (P2, P3, P4, P5 and P6) were simulated for the purpose of $\mathrm{MC}$ calculation. In order to have a reliable MC simulation, the HPGe detector was qualified, the accurate detector geometry and the detector crystal positioning inside the housing were determined by means of radiographic images, $\mathrm{X}$ rays were used to analyse the structure of the detector and $\mathrm{Co}-$ 60 radiography was employed to measure the core of the germanium crystal $^{(42)}$.

Each radionuclide produces a set of photons of well-known energies. When this energy is totally absorbed inside the detector, it is counted in the peak at this energy. The WBC detection geometry does not require taking account of the summation effects, because the distance between the source and the detector is large enough. The MC simulations can compute the response to strict mono-gamma sources, as well as radionuclides with a complex decay scheme. In addition, a correction factor can be evaluated for each specific source or detector geometry and radionuclide.

The parameters of the experimental settings used for measuring the counting efficiency of the PSI$\mathrm{WBC}$ are reported in the PSI extended report on the measurements ${ }^{(43)}$. As mentioned earlier, in a few cases, a number of blocks could not be filled with radioactive inserts, and the phantom was simulated accordingly. The curves describing the system response to monoenergetic photons were simulated as well as its sensitivity to phantom position changes. As shown in Figure 2, the coordinate system used to describe the PSI-WBC setting consisted of a $y$-axis (longitudinal) pointing to the feet of the phantom, an $x$ axis (lateral) pointing sidewards and a $z$-axis pointing upwards. The effect of a change in the position of the phantom on the chair was investigated by simulating

Table 2. Activity and measured energy peaks for the various radionuclides used ${ }^{(43)}$.

\begin{tabular}{|c|c|c|c|c|c|}
\hline Radionuclide & $\begin{array}{l}\text { Measured energy } \\
\text { peaks }(\mathrm{keV})\end{array}$ & $\begin{array}{l}\text { Standard uncertainty } \\
\text { associated with each } \\
\text { efficiency }(\%)\end{array}$ & $\begin{array}{l}\text { Activity per rod for } \\
\text { big blocks }(\mathrm{Bq})\end{array}$ & $\begin{array}{l}\text { Activity per rod for } \\
\text { small blocks (Bq) }\end{array}$ & Reference date \\
\hline \multirow[t]{5}{*}{ Eu-152 } & 121.8 & 3.0 & 78.6 & 40.4 & 1 September 1997 \\
\hline & 344.3 & 2.9 & & & \\
\hline & 778.9 & 3.5 & & & \\
\hline & 964.1 & 3.4 & & & \\
\hline & 1408.0 & 3.2 & & & \\
\hline \multirow[t]{2}{*}{ Ba-133 } & 81.0 & 10.3 & 81.8 & 42.1 & 1 September 1997 \\
\hline & 356.0 & 4.1 & & & \\
\hline \multirow[t]{2}{*}{ Co-60 } & 1173.2 & 2.8 & 150 & 75 & 1 November 2004 \\
\hline & 1332.5 & 2.8 & & & \\
\hline Cs-137 & 661.7 & 3.2 & 83.7 & 43.3 & 1 September 1997 \\
\hline $\mathrm{K}-40$ & 1460.8 & 3.0 & 38.9 & 20.1 & 1 September 1997 \\
\hline
\end{tabular}


Table 3. Number of blocks equipped with radioactive rods during the measurements in phantom configurations P1 to P6 with the five radionuclides used ${ }^{(43)}$.

\begin{tabular}{|c|c|c|c|c|c|c|c|}
\hline \multirow[t]{2}{*}{ Phantom } & \multirow[t]{2}{*}{ Block size } & \multirow[t]{2}{*}{ Total number } & \multicolumn{5}{|c|}{ Number of block equipped with radioactive rods } \\
\hline & & & Eu-152 & Ba-133 & Co-60 & Cs-137 & $\mathrm{K}-40$ \\
\hline \multirow[t]{2}{*}{ P2 } & Big & 21 & 21 & 20 & 21 & 20 & 21 \\
\hline & Small & 6 & 6 & 6 & 6 & 6 & 6 \\
\hline \multirow[t]{2}{*}{ P3 } & Big & 36 & 36 & 36 & 36 & 36 & 36 \\
\hline & Small & 28 & 28 & 28 & 12 & 28 & 28 \\
\hline \multirow[t]{2}{*}{$\mathrm{P} 4$} & Big & 69 & 69 & 69 & 69 & 69 & 69 \\
\hline & Small & 2 & 2 & 2 & 2 & 2 & 2 \\
\hline \multirow[t]{2}{*}{ P5 } & Big & 72 & 70 & 70 & 70 & 70 & 70 \\
\hline & Small & 36 & 36 & 36 & 12 & 33 & 36 \\
\hline \multirow[t]{2}{*}{ P6 } & Big & 90 & 85 & 87 & 80 & 85 & 85 \\
\hline & Small & 40 & 40 & 40 & 12 & 37 & 38 \\
\hline
\end{tabular}

a longitudinal movement (increasing y) and a vertical movement (increasing $z$ ) of the detector.

\section{RESULTS}

\section{Validation of the Monte Carlo code}

The relative differences between the detector responses calculated with GEANT4 and MCNPX were computed with their uncertainties in order to compute a $z$-score. If the values given by both codes were the same, the individual $z$-sores should be approximately Gaussian-distributed with a mean equal to 0 and a standard deviation equal to 1 . The authors observed a mean value of -0.32 and a standard deviation equal to 1.11 . This leads to a $95 \%$ confidence interval for the mean equal to $(-0.37,0.37)$, and therefore the two codes can be considered as being not significantly different. This gives some trust about the authors' implantation of the phantom in the GEANT4 code and its use in the rest of this study.

The comparison between the simulations with a $\mathrm{P} 4$ phantom filled with rods and a P4 phantom uniformly filled with the same activity showed that the efficiency is slightly higher for the homogeneous source. This implies that a calibration with rods leads to an overestimation compared with a calibration with homogeneous activities. Unsurprisingly, the discrepancy increased for low energies. While the difference was a few percentage points for a few hundred kiloelectronvolt, it increased to $10 \%$ at $100 \mathrm{keV}$ and reached $33 \%$ at $40 \mathrm{keV}$.

\section{Experimental data vs. MC-computed monoenergetic response}

The results of the comparison between the experimental and the computational data are presented in Table 4 . The relative biases between measured and
MC-calculated counting efficiencies as well as the $z$ scores are given for the various energy peaks and for the five IGOR phantom configurations used (P2, P3, $\mathrm{P} 4, \mathrm{P} 5$ and P6). An analysis of the $z$-scores shows that most differences are not statistically significant (91\% of the $z$-scores have an absolute value of $\leq 2$ ). The five values that are statistically significant are well distributed across the phantoms (P2, P4, P5 and P6) and across the radioactive sources (Eu-152, Ba-133 and $\mathrm{K}-40$ ). The relative difference between experimental data and MC-computed values averaged over all photon energies, and phantom configurations is only $2 \%$. The $81-\mathrm{keV}$ photon results for Ba-133 show the highest relative bias. The largest observed relative difference is $24 \%$ for the $81-\mathrm{keV}$ photon from Ba- 133 in the $\mathrm{P} 6$ phantom configuration.

Figure 3 compares the experimentally measured counting efficiencies to radionuclides and the MCcalculated counting efficiencies to monoenergetic photons, for phantoms P2 to P6. The measured and the MC-calculated efficiencies agree very well.

\section{Role of phantom positioning}

Table 5 estimates the effect of the position of the detector on the counting efficiency. For a fixed photon energy of $1000 \mathrm{keV}$ and for each of the five phantoms, the authors estimated the counting efficiency at different positions of the detector and then fitted the data by a straight line. The authors did this separately in the $y$ and $z$ directions and used the slope of the line in order to estimate the variation of the counting efficiency per unit of distance; Table 6 presents the same quantity but only for the P4 phantom at set of energies between 100 and $1500 \mathrm{keV}$. The authors observe that the relative variation is not really sensitive to the type of phantom and is $\sim 2 \%$ in the $y$ direction and $\sim 3 \%$ in the $z$ direction. In the P4 phantom, a change 
WHOLE-BODY COUNTER CALIBRATION

Table 4. Deviations between measured and MC-calculated counting efficiencies for the various radionuclides and IGOR phantom configurations used.

\begin{tabular}{|c|c|c|c|c|c|c|c|c|c|c|c|}
\hline \multirow[t]{2}{*}{ Radionuclide } & \multirow{2}{*}{$\begin{array}{l}\text { Peak } \\
(\mathrm{keV})\end{array}$} & \multicolumn{2}{|c|}{$\mathrm{P} 2$} & \multicolumn{2}{|c|}{$\mathrm{P} 3$} & \multicolumn{2}{|c|}{$\mathrm{P} 4$} & \multicolumn{2}{|c|}{ P5 } & \multicolumn{2}{|c|}{ P6 } \\
\hline & & $\begin{array}{c}\text { rel-bias } \\
(\%)\end{array}$ & $z$-score & $\begin{array}{c}\text { rel-bias } \\
(\%)\end{array}$ & $z$-score & $\begin{array}{c}\text { rel-bias } \\
(\%)\end{array}$ & $z$-score & $\begin{array}{c}\text { rel-bias } \\
(\%)\end{array}$ & $z$-score & $\begin{array}{c}\text { rel-bias } \\
(\%)\end{array}$ & $z$-score \\
\hline \multirow[t]{5}{*}{ Eu-152 } & 121.8 & 3 & 0.9 & -5 & -1.6 & -5 & -1.6 & -8 & -2.6 & -3 & -0.8 \\
\hline & 344.3 & 7 & 2.5 & 2 & 0.7 & 4.9 & 1.7 & 1 & 0.2 & 2 & 0.7 \\
\hline & 778.9 & 5 & 1.3 & 2 & 0.6 & 0.9 & 0.3 & 0 & 0.0 & 4 & 1.05 \\
\hline & 964.1 & 5 & 1.3 & 3 & 0.8 & 1 & 0.3 & -1 & -0.3 & 2 & 0.68 \\
\hline & 1408.0 & 8 & 2.5 & 3 & 1.0 & 1.3 & 0.4 & 2 & 0.8 & 3 & 0.98 \\
\hline \multirow[t]{2}{*}{ Ba-133 } & 81.0 & -3 & -0.3 & -18 & -1.7 & -15 & -1.4 & -18 & -1.8 & -24 & -2.28 \\
\hline & 356.0 & 3 & 0.8 & -2 & -0.4 & -2.8 & -0.7 & -3 & -0.7 & -7 & -1.71 \\
\hline \multirow[t]{2}{*}{ Co-60 } & 1173.2 & -3 & -1.2 & -5 & -1.7 & -2.2 & -0.8 & -5 & -1.6 & 0 & 0.07 \\
\hline & 1332.5 & -3 & -0.9 & -5 & -1.9 & -3.1 & -1.1 & -5 & -1.8 & 0 & 0.04 \\
\hline Cs-137 & 661.7 & -1 & -0.2 & -5 & -1.4 & 0.7 & 0.2 & -6 & -1.9 & -2 & -0.62 \\
\hline K-40 & 1460.8 & 1 & 0.4 & 3 & 1.0 & 8.5 & 2.6 & -2 & -0.7 & -6 & -2.00 \\
\hline
\end{tabular}
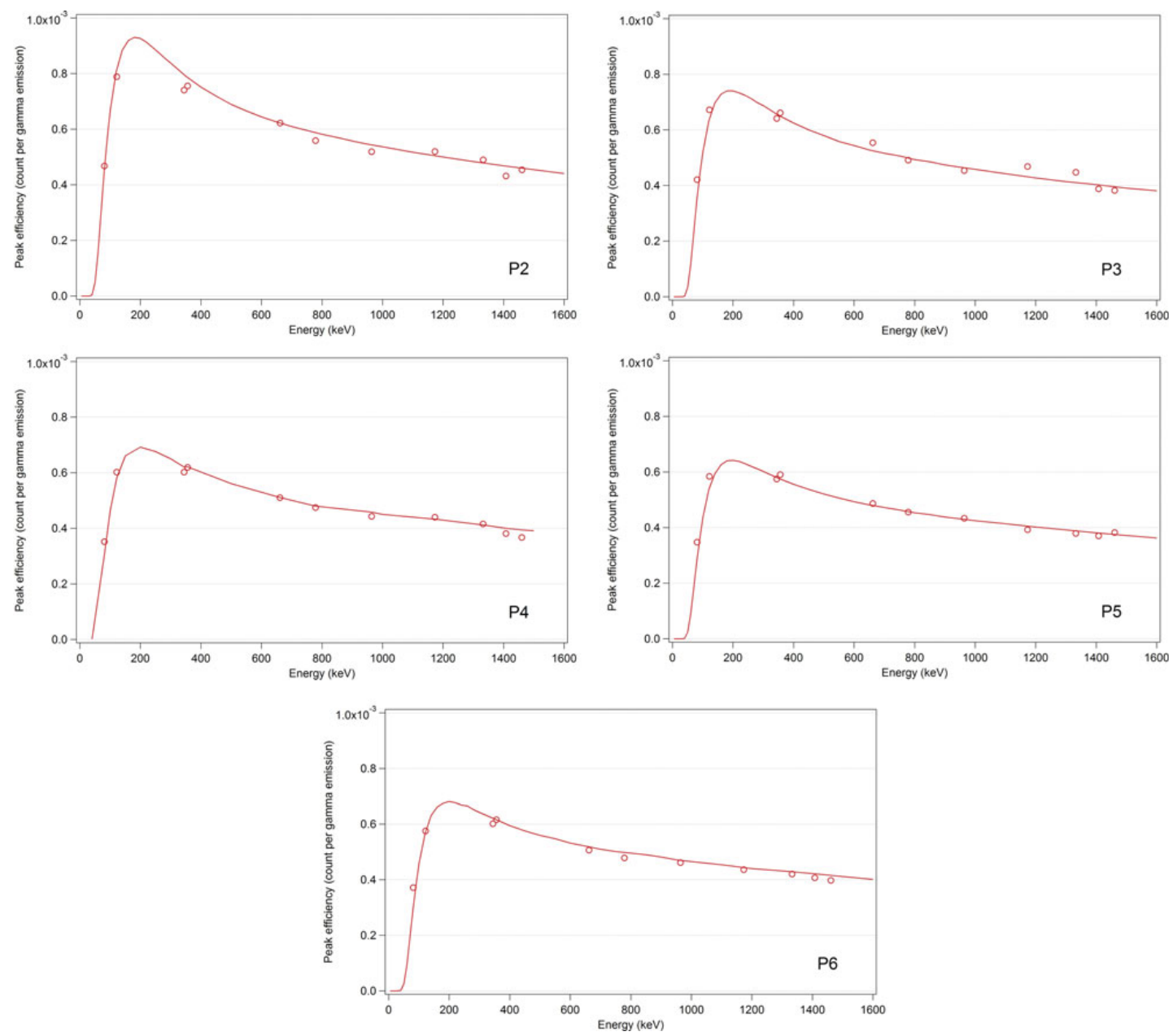

Figure 3. Comparison of measured and MC-calculated counting efficiencies. The measurement points are represented by circles, the MC-calculations of the counting efficiency to monoenergetic photons by a solid line ${ }^{(4,43)}$. 
Table 5. Relative variation of the counting efficiency for a 1-cm displacement of the detector in direction $y\left(\Delta_{\mathrm{y}}\right)$ or $z\left(\Delta_{\mathrm{z}}\right)$ for photon energy of $1000 \mathrm{keV}$.

\begin{tabular}{lll}
\hline Phantom & $\Delta_{\mathrm{y}}(\%)$ & $\Delta_{\mathrm{z}}(\%)$ \\
\hline P2 & -2.3 & -3.2 \\
P3 & -2.6 & -3.4 \\
P4 & -1.9 & -3.0 \\
P5 & -1.6 & -3.1 \\
P6 & -2.8 & -3.5 \\
\hline
\end{tabular}

of photon energy does not significantly change the relative variation.

\section{DISCUSSION}

By and large there is a good agreement between measured and MC-calculated counting efficiencies. Table 4 indicates that the relative deviation between measurement and simulation does not exceed $8.5 \%$. The only exception is Ba-133, which has both the lowest energy and the highest relative uncertainty on the experimental measurements. However, the $z$ scores are globally in the acceptable $(-2,+2)$ range.

The mispositioning of the phantom has a significant effect, and this is coherent with what is observed in other facilities. The results of the effect of the positioning of the detector (or the phantom) presented in Tables 5 and 6 show that there is about a $2 \%$ decrease in the counting efficiency per centimetre along the $y$ axis, and $\sim 3.5 \%$ along the $z$-axis, independently of the phantom configuration and the photon energy. Considering that a $5-\mathrm{cm}$ deviation of phantom positioning is quite realistic, this would impact the sensitivity of the WBC device by $10-20 \%$. It is worth mentioning that this result is specific to a given chairphantom setting; a recent study by Krstic and Nikezic ${ }^{(33)}$ has shown that a more compact setting, where the trunk and the legs of the phantoms are perpendicular, leads to a translation effect of the detector along the $y$-axis that is not significant, whereas Genicot et al $^{(32)}$ reported a $1 \%$ decrease per centimetre along the $y$-axis when the phantom is in a bed (lying) position but only $\sim 0.5 \%$ per centimetre in a tilted chair position, and a $1.3 \%$ decrease in the counting efficiency per centimetre along the $x$-axis. The decrease of the counting efficiency along the $z$ axis reported by these authors is $2.4 \%$ per centimetre.

MC simulation appears to be suitable for the calibration of WBCs with phantoms in complex settings. The usefulness of voxel phantoms, however, may be questioned since it may improve the accuracy of the shape and position of the organs in the body. But this level of accuracy is not that relevant considering the uncertainties related to a mispositioning of the phantom itself. While some authors consider them
Table 6. Relative variation of the counting efficiency for a 1-cm displacement of the detector in direction y $\left(\Delta_{y}\right)$ or $z\left(\Delta_{z}\right)$ for the $\mathrm{P} 4$ phantom.

\begin{tabular}{lcc}
\hline Energy $(\mathrm{keV})$ & $\Delta_{\mathrm{y}}(\%)$ & $\Delta_{\mathrm{z}}(\%)$ \\
\hline 100 & -1.8 & -2.6 \\
200 & -1.9 & -2.8 \\
500 & -2.0 & -2.8 \\
1000 & -1.9 & -3.0 \\
1500 & -1.9 & -3.0 \\
\hline
\end{tabular}

'significant tools' for the calibration of $\mathrm{WBC}^{(35)}$, others believe that 'for in vivo counting in the context of radiation protection, efficiencies can be deduced with sufficient accuracy from measurements or simulations of simple phantoms ${ }^{3(5)}$. Guardini and Ferrari ${ }^{(10)}$ argue that the accuracy in geometry representation improved by voxel models is only one contributor to the overall accuracy of a WBC measurement and not the most important. Other parameters are more critical, such as precision of the radiation interaction data libraries, the dose conversion factors and the knowledge of the source space and energy distributions. They concluded by saying that 'a voxel representation of a standard man does not imply dramatic variations compared with the stylised models, apart from some internal dosimetry applications, on a correct radiological protection assessment'.

The choice of a phantom should always be related to its use. In this study, the authors' goal was to improve instrument calibration. Therefore, the authors showed that the IGOR phantom is very sensitive to few centimetre displacements that are unavoidable in practice. This is coherent with the findings of De Carlan et al. ${ }^{(47)}$ who found a difference of $<10 \%$ between the use of the voxel IGOR phantom and the voxel human body phantom (Zubal) for the calibration of whole-body counters, confirming that IGOR could be considered a realistic representation of a human. This may not be sufficient for evaluating influence factors in the case of heterogeneous internal contamination where voxel phantoms may well be invaluable ${ }^{(48)}$.

\section{CONCLUSION}

The authors used the MC code GEANT4 in order to simulate a WBC measuring a set of IGOR phantoms. The authors showed that the MC simulations give results that are consistent with the experimental measurements with relative deviations well below $10 \%$. The only exception was for the Ba-133 source that had a large experimental uncertainty. The investigation also showed that a $1-\mathrm{cm}$ error in positioning leads to $2-3 \%$ in activity estimation. Considering 
that a $5-\mathrm{cm}$ deviation on the positioning of the phantom is a realistic scenario, this would impact the sensitivity of the WBC device by $>10 \%$.

\section{FUNDING}

This work was funded by grant by the Swiss Federal Nuclear Safety Inspectorate (ENSI).

\section{REFERENCES}

1. Alderson, S. W., Lanzl, L. S., Rollins, M. and Spire, J. An instrumented phantom system for analog computation of treatment plans. Amer. J. Roentgen. 87, 185-195 (1962).

2. Griffith, R. V., Dean, P. N., Anderson, A. L. and Fisher, J. C. A tissue equivalent torso phantom for intercalibration of in vivo transuranic nuclide counting facilities. Advances in Radiation Protection Monitoring, STI/ PUB/494 (IAEA), 4493-4504 (1979).

3. American National Standards Institute. Specifications for the bottle manikin absorption phantom. ANSI/HPS N13.35-1999, American National Standards Institute (ANSI) (2000).

4. Kovtun, A. N., Firsanov, V. B., Fominykh, V. I. and Isaakyan, G. A. Metrological parameters of the unified calibration whole-body phantom with gamma-emitting radionuclides. Radiat. Prot. Dosim. 89, 239-242 (2000).

5. Breustedt, B., Paetz gen Schieck, H., Schicha, H. and Eschner, W. Calibration of a whole body counter using Monte Carlo methods [Article in German]. Z. Med. Phys. 14(2), 85-94 (2004).

6. Kinase, S., Takagi, S., Noguchi, H. and Saito, K. Application of voxel phantoms and Monte Carlo method to whole-body counter calibration. Radiat. Prot. Dosim. 125(1-4), 189-193 (2007).

7. Cristy, M. Mathematical phantoms representing children of various ages for use in estimates of internal dose. ORNL/NUREG/TM-367, NUREG/CR-1159 (Oak Ridge National Laboratory) (1980).

8. Eckerman, K. F., Cristy, M. and Ryman, J. C. The ORNL mathematical phantom series. (Oak Ridge National Laboratory) (1996). Available on http:// ordose.ornl.gov/resources/Mird.pdf (accessed 3 April 2013).

9. Kramer, R., Zankl, M., Williams, G. and Drexler, G. The calculation of dose from external photons exposures using human phantoms and Monte Carlo methods: Part I: The male (Adam) and female (Eva) adult mathematical phantom. GSF-Report S-885 reprint, German Institute of Radiation Protection (1986).

10. Gualdrini, G. and Ferrari, P. A review of voxel model development and radiation protection applications at ENEA. Radiat. Prot. Dosim. 140(4), 383-390 (2010).

11. International Commission on Radiological Protection. Adult reference computational phantoms. ICRP Publication 110 (Pergamon Press) (2009).

12. Dimbylow, P. J. FDTD calculations of the whole-body averaged $S A R$ in an anatomically realistic voxel model of the human body from $1 \mathrm{MHz} 1 \mathrm{GHz}$. Phys. Med. Biol. 42, 479-490 (1997).
13. Xu, X. G., Chao, T. C. and Bozkurt, A. VIP-Man: an image-based whole-body adult male model constructed from color photographs of the visible human project for multi-particle Monte Carlo Calculations. Health Phys. 78, 476-486 (2000).

14. Zankl, M. and Wittmann, A. The adult male voxel model 'Golem' segmented from whole body CT patient data. Radiat. Environ. Biophys. 40, 153-622 (2001).

15. Saito, K., Wittmann, A., Koga, S., Ida, Y., Kamei, T., Funabiki, J. and Zankl, M. Construction of a computed tomographic phantom for a Japanese male adult and dose calculation system. Radiat. Environ. Biophys. 40, 69-76 (2001).

16. Kramer, R., Khoury, H. J., Vieira, J. W. and Lima, V. J. M. MAX06 and FAX06: update of two adult human phantoms for radiation protection dosimetry. Phys. Med. Biol. 51, 3331-3346 (2006).

17. Kramer, R., Khoury, H. J., Vieira, J. W., Loureiro, E. C. M., Lima, V. J. M., Lima, F. R. A. and Hoff, G. All about FAX: a Female Adult voXel phantom for Monte Carlo calculation in radiation protection dosimetry. Phys. Med. Biol. 49, 5203-5216 (2004).

18. Lee, C. T. and Lee, J. The Korean adult male voxel model 'Korman' segmented form magnetic resonance images. Med. Phys. 31, 1017-1022 (2004).

19. Mofrad, F. B., Zoroofi, R. A. and Tehrani-Fard, A. A. Statistical construction of a Japanese male liver phantom for internal radionuclide dosimetry. Radiat. Prot. Dosim. 141(2), 140-148 (2010).

20. Piegl, L. and Tiller, W. The NURBS Book. (Springer) (1997).

21. Lee, C., Lodwick, D., Hasenauer, D., Williams, J. L., Lee, C. and Bolch, W. E. Hybrid computational phantoms of the male and female newborn patient: NURBSbased whole-body models. Phys. Med. Biol. 52, 3309-3333 (2007).

22. Kramer, R., Cassola, V. F., Khoury, H. J., Vieira, J. W., de Melo Lima, V. J. and Robson Brown, K. FASH and MASH: female and male adult human phantoms based on polygon mesh surfaces: II. Dosimetric calculations. Phys. Med. Biol. 55, 163-189 (2010).

23. Kim, C. H., Jeong, J. H., Bolch, W. E., Cho, K.-W. and Hwang, S. B. A polygon-surface reference Korean male phantom (PSRK-Man) and its direct implementation in Geant4 Monte Carlo simulation. Phys. Med. Biol. 56, 3137-3161 (2011)

24. Consortium of Computational Human Phantoms (CCHP). List of human phantoms. Available on http:/ www.virtualphantoms.org. (accessed 3 April 2013). CCHP.

25. Fasso, A., Ferrari, A., Ranft, J. and Sala, P. R. FLUKA. a multi-particle transport code. CERN-2005-10, INFN/ TC_05/11, SLAC-R-773, European Organization for Nuclear Research (CERN) (2005).

26. Park, S., Lee, J. K. and Lee, C. Development of a Korean adult male computational phantom for internal dosimetry calculation. Radiat. Prot. Dosim. 121(3), 257-264 (2006).

27. Zhang, B. Q., Ma, J. Z., Liu, L. Y. and Cheng, J. P. CNMAN: a Chinese adult male voxel phantom constructed from color photographs of a visible anatomical data set. Radiat. Prot. Dosim. 124, 130-136 (2007).

28. Pelowitz, D. B. Ed. MCNPX User's Manual. LACP-050369 (Los Alamos Laboratory) (2005).

29. Zhang, B., Mille, M. and Xu, X. G. An analysis of dependency of counting efficiency on worker anatomy for in 


\section{F. O. BOCHUD ET AL.}

vivo measurements: whole-body counting. Phys. Med. Biol. 53:3463-3475 (2008).

30. Schläger, M. Comparison of various anthropomorphic phantom types for in vivo measurements by means of Monte Carlo simulations. Radiat. Prot. Dosim. 144, 384-388 (2011).

31. Capello, K., Kedzior, S. and Kramer, G. H. Voxel phantoms: the new ICRP computational phantoms: how do they compare? Health Phys. 102, 626-630 (2012).

32. Bento, J., Barros, S., Teles, P., Neves, M., Gonçalves, I., Corisco, J. and Pedro Vaz, P. Monte Carlo simulation of the movement and detection efficiency of a whole-body counting system using a BOMAB phantom. Radiat. Prot. Dosim. 148(4), 403-413 (2012b).

33. Krstic, D. and Nikezic, D. Efficiency of whole-body counter for various body size calculated by MCNP5 software. Radiat. Prot. Dosim. 152(1-3), 179-183 (2012).

34. Kramer, R., Vieira, J. W., Khoury, H. J., Lima, F. R. A. and Fuelle, D. All about MAX: a male adult voxel phantom forMonte Carlo calculations in radiation protection dosimetry. Phys. Med. Biol. 48, 1239-1262 (2003).

35. Takahashi, M., Kinase, S. and Kramer, R. Evaluation of counting efficiencies of a whole-body counter using Monte Carlo simulation with voxel phantoms. Radiat. Prot. Dosim. 144(1-4), 407-410 (2011).

36. Brun, R., Bruyant, F., McPherson, A. C. and Zanarini, P. GEANT detector description and simulation tool. CERN Report DD/EE/84-1, Genf, European Organization for Nuclear Research (CERN) (1994).

37. Bhati, S., Patni, H. K., Ghare, V. P., Singh, I. S. and Nadar, M. Y. Monte Carlo calculations for efficiency calibration of a whole-body monitor using BOMAB phantoms of different sizes. Radiat. Prot. Dosim. 148(4), 414-419 (2012).

38. Bento, J., Barros, S., Teles, P., Vaz, P. and Zankl, M. Efficiency correction factors of an ACCUSCAN whole- body counter due to the biodistribution of $134 \mathrm{Cs}, 137 \mathrm{Cs}$ and 60Co. Radiat. Prot. Dosim. (2012a) PMID 23188813.

39. Genicot, J. L., Koukouliou, V. and Carinou, E. Monte Carlo calculations applied to the parametrical studies in a whole body counter. Radiat. Prot. Dosim. 128(1), 49-61 (2008).

40. Swiss Federal Office of Public Health. Dosimétrie des personnes exposées aux radiations dans l'exercice de leur profession en Suisse. Rapport annuel 2011. (FOPH) (2012).

41. Boschung, M. The high purity germanium detector wholebody monitor at PSI. Radiat. Prot. Dosim. 79(1-4), 481-484 (1998).

42. Mayer, S., Boschung, M., Meier, K., Laedermann, J.-P. and Bochud, F. O. Characterisation of the PSI whole body counter by radiographic imaging. Radiat. Prot. Dosim. 144(1-4), 398-401 (2011).

43. Chetelat, N. Laborjournal IGOR-Messungen mit HPGe Ganzkörperzähler am PSI. (Paul Scherrer Institut) (2010).

44. Agostinelli, S., Allison, J., Amako, K. et al. Geant4-a simulation toolkit Original Research Article. Nucl. Instrum. Method. Phys. Res. A 506(3), 250-303 (2003).

45. Allison, J., Amako, K. and Apostolakis, J. et al. Geant4 developments and applications. IEEE Trans. Nucl. Sci. 53(1), 270-278 (2006).

46. Laedermann, J.-P. and Décombaz, M. Simulation of nuclear decay. Appl. Radiat. Isot. 52(3), 419-425 (2000).

47. de Carlan, L., Roch, P., Blanchardon, E. and Franck, D. Application of voxel phantoms in whole-body counting for the validation of calibration phantoms and the assessment of uncertainties. Radiat. Prot. Dosim. 125(1-4), 477-482 (2007).

48. Lamart, S., Blanchardon, E., Molokanov, A., Kramer, G. H., Broggio, D. and Franck, D. Study of the influence of radionuclide biokinetics on the efficiency of in vivo counting using Monte Carlo simulation. Health Phys. 96(5), 558-567 (2009). 